\title{
Chapter
}

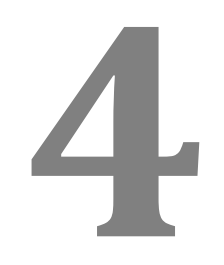

\section{RECENT ADVANCES IN MICROFLUIDIC DIAGNOSTIC AND TREATMENT SYSTEMS FOR ASSISTED REPRODUCTIVE TECHNOLOGIES IN DEVELOPING COUNTRIES}

Koji Matsuura, ${ }^{*}$ Saori Nishina, and Yuka Asano

Faculty of Biomedical Engineering, Department of Engineering, Okayama University of Science, Japan 


\section{Contents}

4.1. INTRODUCTION

4.2. CURRENT INFERTILITY TREATMENTS AND TECHNICAL SOLUTIONS FOR MALE INFERTILITY IN DEVELOPING COUNTRIES ................................................................. 96

4.3. SMARTPHONE-BASED OPTICAL SYSTEMS .......................................................................... 97

4.4. MICROFLUIDIC CHANNEL-BASED DEVICES FOR SEMEN DIAGNOSES AND

MALE INFERTILITY TREATMENTS....................................................................................100

4.5. PAPER-BASED DEVICES FOR SEMEN DIAGNOSIS...............................................................104

4.6. DISCUSSION AND SATISFACTION OF CRITERIA FOR SEMEN

CHARACTERISTICS....................................................................................................................108

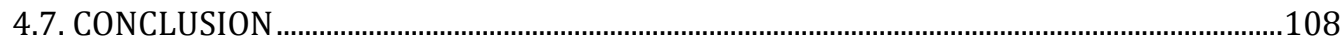

ACKNOWLEDGEMENTS ..............................................................................................................108

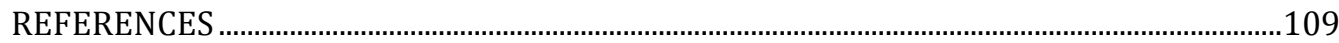




\subsection{INTRODUCTION}

Male infertility contributes to approximately half of all infertility cases, and approximately 50 million couples suffer from infertility globally [1-3]. Rates of male infertility range from $20 \%-70 \%$ [1], and in sub-Saharan African countries, infertility affects up to $40 \%$ of men partly ue to high rates of sexually transmitted diseases [1,2]. In low-resource countries, poor clinical access precludes examinations of sperm characteristics in infertile males [4-6]. In assisted reproductive technology (ART) laboratories, male fertility is commonly assessed by using a microscope to determine numbers of motile human sperm, degrees of sperm motility and sperm morphology. The World Health Organization (WHO) criteria for semen characteristics were updated in 2010 [1,2,7], and those for sperm concentrations, normal morphologies and progressive motility were 15 million cells, $5 \%$ and $40 \%$, respectively. Semen that satisfied the WHO criteria are washed and inseminated into the uterus using the minimally invasive procedure intrauterine insemination (IUI) [8]. In contrast, when sperm concentrations are below the criteria (oligozoospermia) or abnormal sperm motility and / or sperm structure (tetratozoospermia) are found, males are diagnosed with subfertility. These couples are then treated using conventional in vitro fertilization (IVF) and / or intracytoplasmic sperm injections (ICSI) [2,9]. Causes of subfertility include sexual disorders, testicular defects in sperm production, endocrinopathies that affect spermatogenesis and defects in sperm transportation [9]. When no sperm are observed in semen (azoospermia), microsurgical epidydimal sperm aspiration (MESA) and testicular sperm extraction (TESE) are the standard methods used for sperm retrieval [10]. However, ICSI, MESA and TESE are expensive treatments and are not affordable at assisted reproductive technologies (ART) clinics in developing countries. In particular, finding sperm using MESA and TESE methods is associated with high labour costs. Furthermore, people in developing countries cannot easily consult ART clinics [4]. Hence, more convenient technologies for the treatment of male infertility and monitoring of sperm concentration, motility and DNA integrity are desired, and technological improvements related to semen characteristics have been investigated for improved ART. However, monitoring of human sperm health in resourcelimited or remote settings is challenging due to (1) high capital costs (equipment for currently accepted procedural standards) and (2) complexity of currently accepted procedures for simultaneous measurements of human sperm concentration and motility. Fertility treatments and potentially low-cost microfluidic diagnostic devices are shown in Figure 1. 


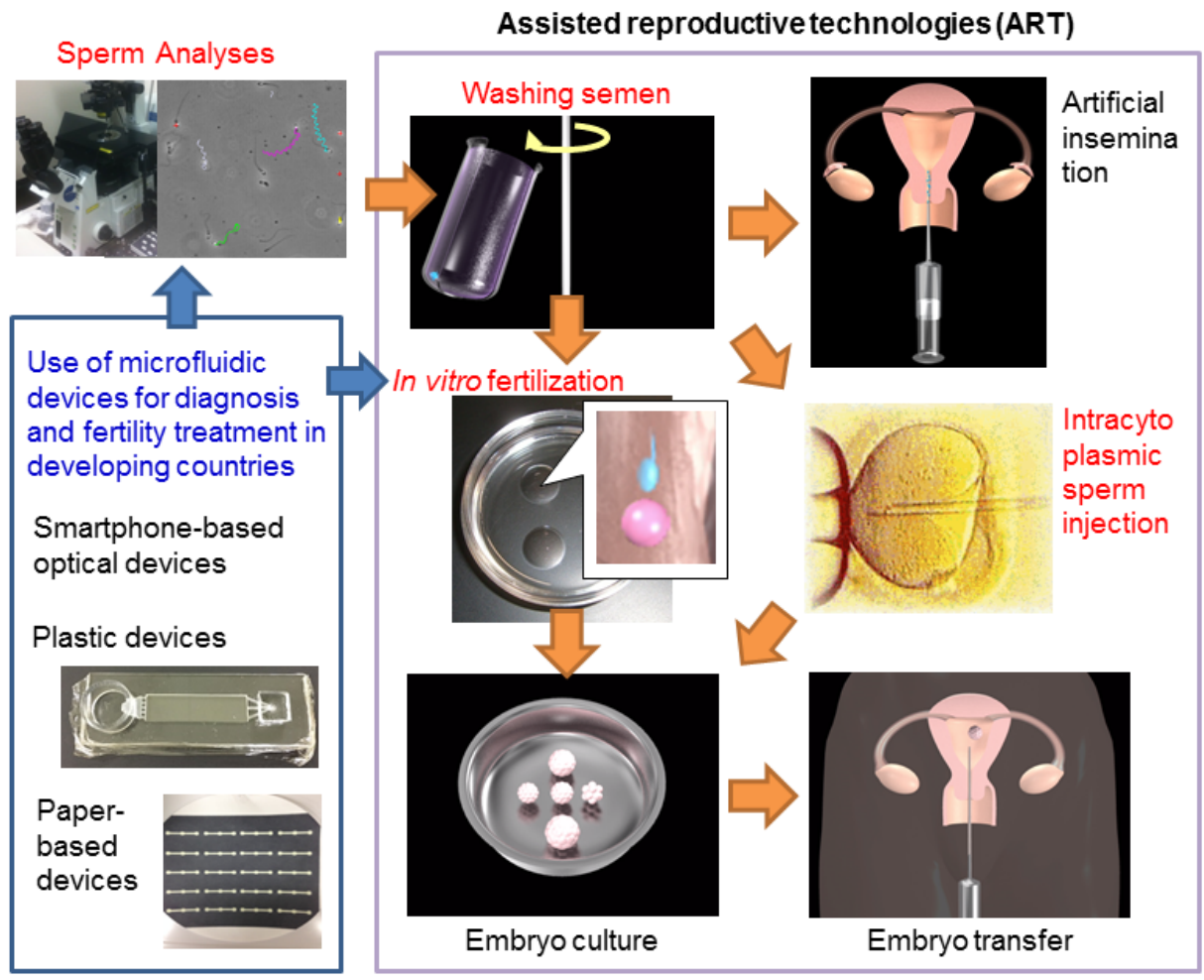

Figure 1. Schematic presentation of the ART procedure and the concept of this review. Microfluidics and smartphone-based optical devices can facilitate sperm analyses, semen washing and IVF.

\subsection{CURRENT INFERTILITY TREATMENTS AND TECHNICAL SOLUTIONS FOR MALE INFERTILITY IN DEVELOPING COUNTRIES}

Inhorn et al. calculated numbers of IVF clinics per 10 million persons and reported fewer than 20 IVF clinics in sub-Saharan Africa in 2010 and fewer still in other regions [4,11]. Although Japan and India have 600 and 500 IVF clinics, respectively, these statistics have not been generated in some Central Asian counties, where numbers of IVF clinics are also few. Hence, ART services remain inaccessible in many parts of the world, particularly in sub-Saharan Africa [4]. Thus, to avoid the extreme costs of infertility treatments, people who desire ART services choose low-cost IVF (LCIVF) programs or global cross-border reproductive care (CBRC) by traveling to low-cost ART affordable countries [4]. IVF techniques and costs are strongly related to infertility/subfertility conditions and countries, and single cycles of IVF cost 9500, 6400 and 1300 USD in the United States, Hong Kong and Pakistan, respectively [4]. The LCIVF program aimed to reduce IVF treatment costs to 
200 euros per cycle by simplifying embryo culture methods and eliminating the need for high-end equipment $[4,12]$. However, LCIVF does not currently replace high-cost ICSI and IVF, which are designed to overcome male infertility [4], because user-friendly technologies for male fertility treatment and diagnostics remain satisfactory for couples in developing counties. Hence, technological breakthroughs are required for robust, low-cost male infertility treatments, because male infertility rates in developing counties remain a serious problem despite the development of current LCIVF programs [13].

No practical protocols have been devised for LCIVF programs, and clinical routines for improved diagnosis and management of ART processes have not been standardized for resource-limited circumstances in developing countries $[4,14]$. We would like to suggest microfluidic technology that can be used without expensive instruments, because these microfluidic devices for sperm sorting or determinations of sperm concentrations have potential as fertility treatments and can be used in the absence of skilled embryologists. These devices could be beneficial if they were optimized to reduce labour costs for patients. Microfluidic devices will also contribute to diagnostic measurements of sperm concentrations, motility analyses and DNA integrity analysis. Candidate methods for LCIVF include microscopic, colorimetric and electronic analytical systems using smartphone-based optical systems, microfluidic systems and paper-based systems. In this chapter, we summarized recent technologies for infertility treatments and portable low-cost point-of-care (POC) systems and made comparisons with current ART procedures.

\subsection{SMARTPHONE-BASED OPTICAL SYSTEMS}

The development of low-cost microfluidic devices for POC is closely linked with emergency capabilities of smartphones, which were released by Apple and Google in 2007 and have since been incorporated into peoples' lives $[15,16]$. Although broadband penetration is low in developing countries, remaining below $10 \%$ in South Africa, smartphones have penetrated approximately $40 \%$ of these areas $[15,16]$. Hence, based on information and communication technologies (ICT) infrastructure conditions in developing countries, portable smartphones are the best suited terminals for combinations of diagnostics and medical instruments, such as microscopes and electrocardiograms (ECG) $[17,18]$. Accordingly, smartphones have been used individually and with other components or devices to monitor cardiovascular disease [17]. Microscopic POC devices using smartphones were also developed during this decade. Specifically, Tujin et al. conducted a feasibility study of microscopy-based diagnostic services to find microbes in body fluid using conventional staining techniques, such as Giemsa [18]. Moreover, Wei et al. demonstrated the use of fluorescence microscopic diagnostic systems with smartphones to visualize single bacteria or viruses, and developed an 
immunostaining method and optical system using a cell phone attachment [19]. Pathogenic and non-pathogenic bacterial concentrations of greater than $10^{4} \mathrm{CFU}$ per millilitre of ejaculate are considered significant bacteriospermia. Hence, bacterial detection technologies that can be used with semen before IUI and IVF will contribute to fertility, especially in developing countries [20-23]. Systems comprising smartphone and microfluidic devices [24] and paper-based devices [25] have also been proposed. In particular, spores of Bacillus anthracis, the causative agent of anthrax, were detected in an incubation chamber of a developed microfluidic channel using a smartphone microscope without the use of staining or PCR methods [24]. Moreover, Salmonella concentrations were evaluated by detecting light scatter intensities at $475 \mathrm{~nm}$ from the centres of paper microfluidic channels [25]. Hence, improvements of smartphone-based detection technologies using chemical staining methods and optical systems will likely be applied to ART and LCIVF.

Low-cost microscopic technologies can be used to perform satisfactory sperm motility analyses, and current IVF procedures are used to evaluate sperm characteristics in ART clinics. Specifically, sperm motility (percentage of motile sperm) and trajectories are confirmed using standard protocols for computer-assisted semen analysis (CASA) systems, and the semen characteristics are evaluated according to WHO criteria as described above. Accordingly, Garrett et al. demonstrated a relationship between average straight-line velocity of motile sperm and pregnancy rates in subfertile couples [26]. Mortimer et al. also showed that CASA works well with washed human semen, which typically has very high motility and minimal contamination with other cells and debris [27]. Hence, these accurate CASA determinations of sperm concentrations and percentages of motile or progressively motile sperm indicate high utility of this tool for fertility assessments [27].

In developing countries, the price of a CASA system (usually over 10,000 USD) is too high to be used in ART clinics. Thus, low-cost, portable devices for quantitative sperm analysis have been developed during the last decade [2834]. Optical systems using these devices in sperm motility analyses are presented in Figure 2. In 2009, a mobile phone-based clinical microscopic system was used to detect tuberculosis via fluorescence imaging in resource-limited regions [28]. Subsequently, a light-weight, portable microscopic system with a lens-free holographic on-chip microscope was proposed in 2010 [29]. Hence, lens-free imaging systems are preferred for low-cost, lightweight POC systems (Figure 2A, 2B, and 2C). 
A

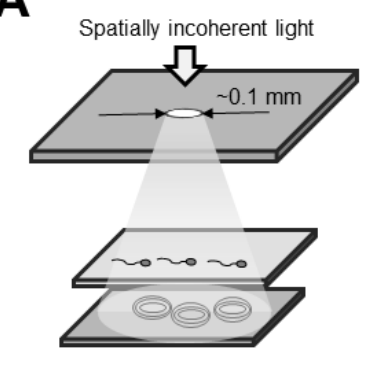

B

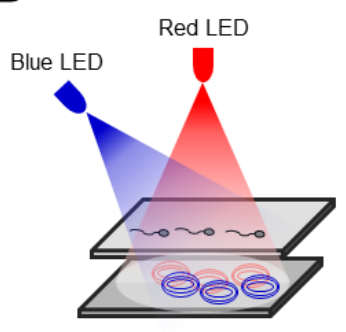

D

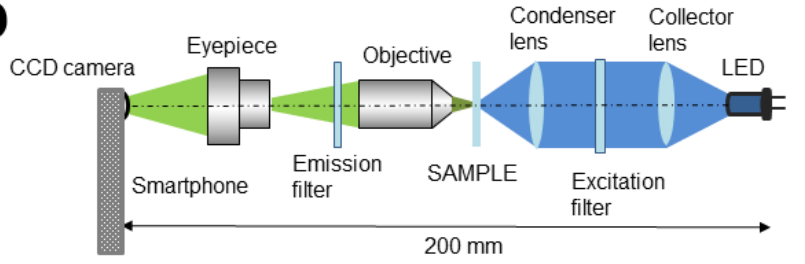

E
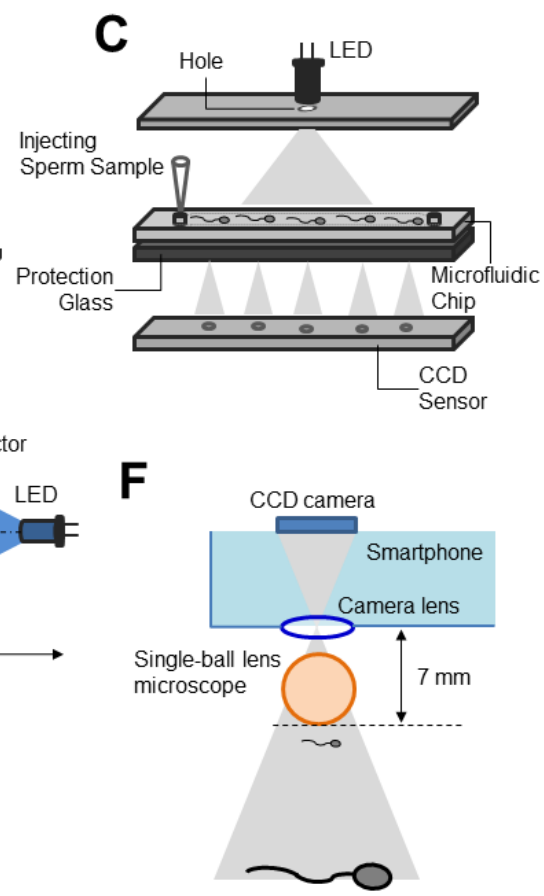

Figure 2. Comparison of optical systems for optical sperm imaging systems;

(A) Lens-free holographic on-chip microscope [29], (B) 3D tracking system for human sperm [30], (C) Lens-free shadow-based CCD imaging system [31], (D) Mobile or smartphone-based clinical microscopic system to detect fluorescence from samples

[28], (E) Smartphone-based optical device using diffraction patterns [32],

(F) Smartphone-based sperm observation system using a single ball lens [33].

In 2012, Su et al. developed a three-dimensional (3D) tracking system for human sperms by illuminating spermatozoa at two different angles using two partially coherent LEDs with different wavelengths (red and blue LEDs at $625 \mathrm{~nm}$ and $470 \mathrm{~nm}$, respectively) [30]. Using this device, sperm motility and trajectories of motile sperm could be recorded. Subsequently, a lens-free, shadow-based, charge-coupled device (CCD) imaging system with a microfluidic chip was reported in 2011, and oligozoospermic and oligospermaesthenic cases were detected using trajectory analyses and sperm sorting with this system [31]. In 2015, Im et al. developed a smartphone-based optical device that exploits diffraction patterns generated by microbeads following binding to biological targets, and detected human papillomavirus DNA. [32] To apply smartphone-based, low-cost microscopic devices, a single-ball lens of $0.8 \mathrm{~mm}$ diameter was purchased for less than 10 USD and was installed on a smartphone to analyse motile sperm concentrations [33]. Kobori et al. correlated measurements of motility from a smartphone-based 
device, conventional CASA [33]. Moreover, the Apple iOS application iSperm uses an optical lens attachment and is commercially available for animal husbandry applications [34]. Although motility can be analysed using smartphone-based, low-cost optical systems, these systems lack algorithms for trajectory analyses, which are often costly. Hence, whereas smartphone applications readily introduce the newest technology, possible obstacles to diffusion of smartphone-based observation systems include penetration of broadband into developing countries, which will lead to effective communication between patients and hospitals, regulation of personal information and distribution of accessories such as chip and paper devices.

\subsection{MICROFLUIDIC CHANNEL-BASED DEVICES FOR SEMEN DIAGNOSES AND MALE INFERTILITY TREATMENTS}

Microfluidic chip technologies, known as lab-on-a-chip (LOC), have been extended to POC applications, such as diagnosis of cardiovascular and infectious diseases with high death rates [35-38], and have been commercialized [35]. These LOCs comprise combinations of expensive analytical instruments that are used to evaluate cell and molecule concentrations or functions using photometry or electrochemistry. These microfluidic devices can be used by pipetting sample liquid without complex fluid manipulation systems, such as electric pumps and bulbs, leading to the advantages of simplicity and low cost. Since the 1990s, microfluidic devices for sperm counts and motility analyses have been investigated by Kricka et al. [39], and microfluidic POC systems for semen diagnostics sorting motile sperms or on-chip IVF using LOC have been reported [40-51]. Moreover, in addition to motile sperm separation, fluid flow- and viscosity-associated chemotaxis and mechanotaxis of mammalian sperm were investigated using microfluidic channels [45]. When mouse sperm were sorted using space-constrained lateral microfluidic channels, effective channel lengths were greater than $15 \mathrm{~mm}$ for sperm sorting with high velocity, and were effective for separation from non-motile sperm and debris [49]. Chen et al. also developed a microfluidic device to evaluate motile sperm concentration and motility [50]. This chip device has two channels for application to semen and buffer, and after movement of motile sperm to the buffer channel, sperm samples in both channels are centrifuged. This device may enable sperm quality assessments without a microscope or a skilled embryologist at resource-limited clinics. Taken together, these studies indicate that LOC devices will improve the accuracy of fertility tests and male infertility treatments [51].

Both POC diagnostic devices and clinical infertility treatment devices require FDA approval for material and production processes, but they are applicable to both sperm and oocyte manipulations. Among LOC devices, microfluidic sperm 
sorting (MFSS) chips have been developed and marketed for motile sperm sorting and use two gravity-driven laminar flows across a microfluidic channel of 50-200 $\mu \mathrm{m}$ [52-61]. Fluids flowing through the semen inlet (A) and the medium inlet (B) move parallel to each other and exit through respective outlets $(A \rightarrow D$ and $B \rightarrow C)$ [53]. Sperm are then sorted on the basis of their ability to swim across the streamline into the medium stream, and only motile sperm are recovered from outlet C. Using MFSS devices, embryologists can perform a 1-step sorting protocol without centrifugation, and processing can be completed within 30 min [40,57-61]. Moreover, DNA fragmentation was reduced and sperm with high linear velocities (LV) were effectively sorted $[58,60]$. Hence, commercialized MFSS devices can be used with clinical semen processing protocols for efficient ICSI and IVF. Recent MFSS studies suggest that DNA fragmentation rates in sorted sperm are lower following MFSS than following the centrifugation and swim-up procedure [61].
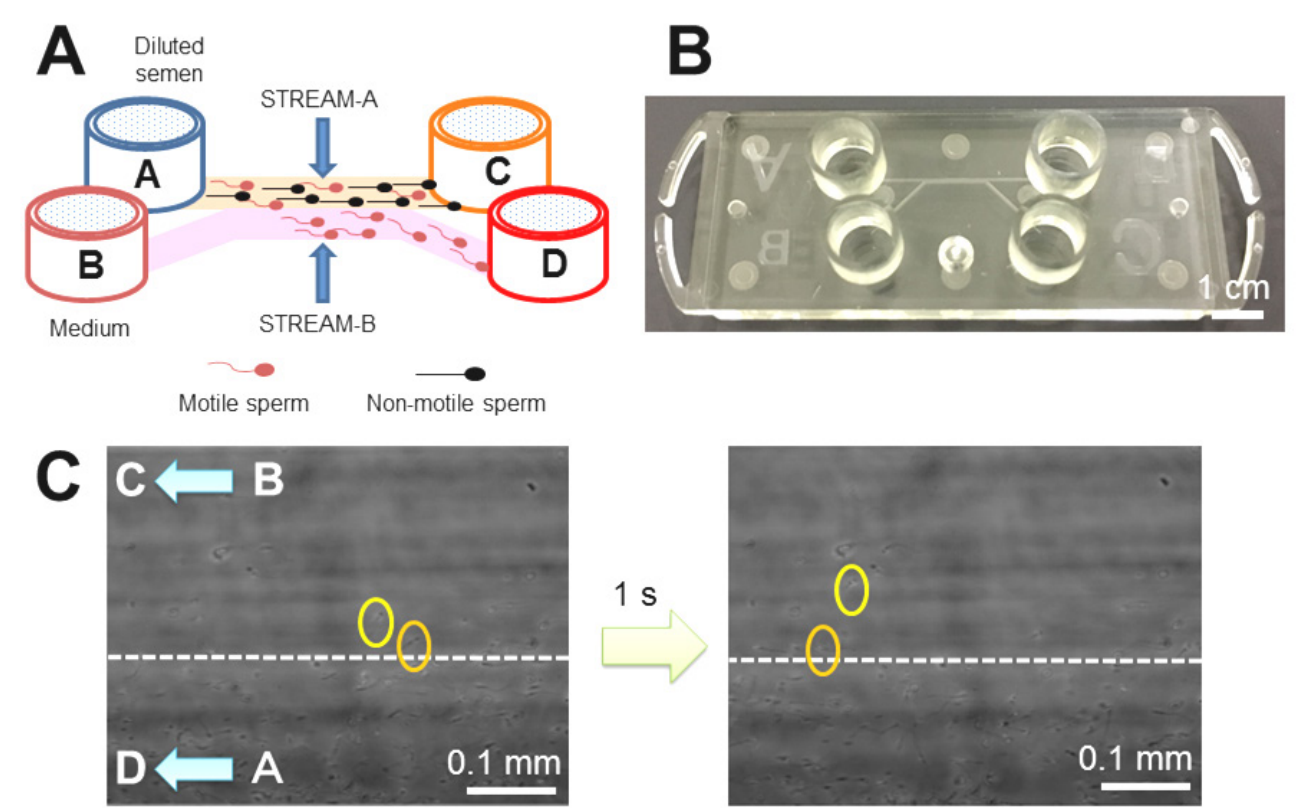

Figure 3. (A) Schematic of the mechanism of microfluidic sperm sorting devices;

(B) Chip device made of cyclo-olefin polymer (Menicon Co. Ltd.); (C) Microscope images during sperm sorting; Yellow and orange circles show human sperm swimming across the interface of the two laminar flows.

Although MFSS-sorted sperm are suitable for ICSI, sorted sperm concentrations are currently insufficient for IUI. This disadvantage of low recovered motile sperm concentrations follows dilution of semen to control viscosity for laminar flow adjustments. Hence, motile sperm were sorted using 
microfluidic channels equipped with micro-pore filters (pore size: $4-20 \mu \mathrm{m}$ ) to separate non-motile sperm from those that swim up in a vertical direction, and motility and morphology of sorted sperm were greater than in unsorted sperm $[62,63]$. Because sorted motile sperm concentrations have not been discussed previously, it remains unclear whether this device can recover motile sperm concentrations sufficiently for IUI [63]. Sperm sorting microfluidic devices that do not require centrifugation may be applied to conventional IUI after technological improvements that sort motile sperm without reducing motility.

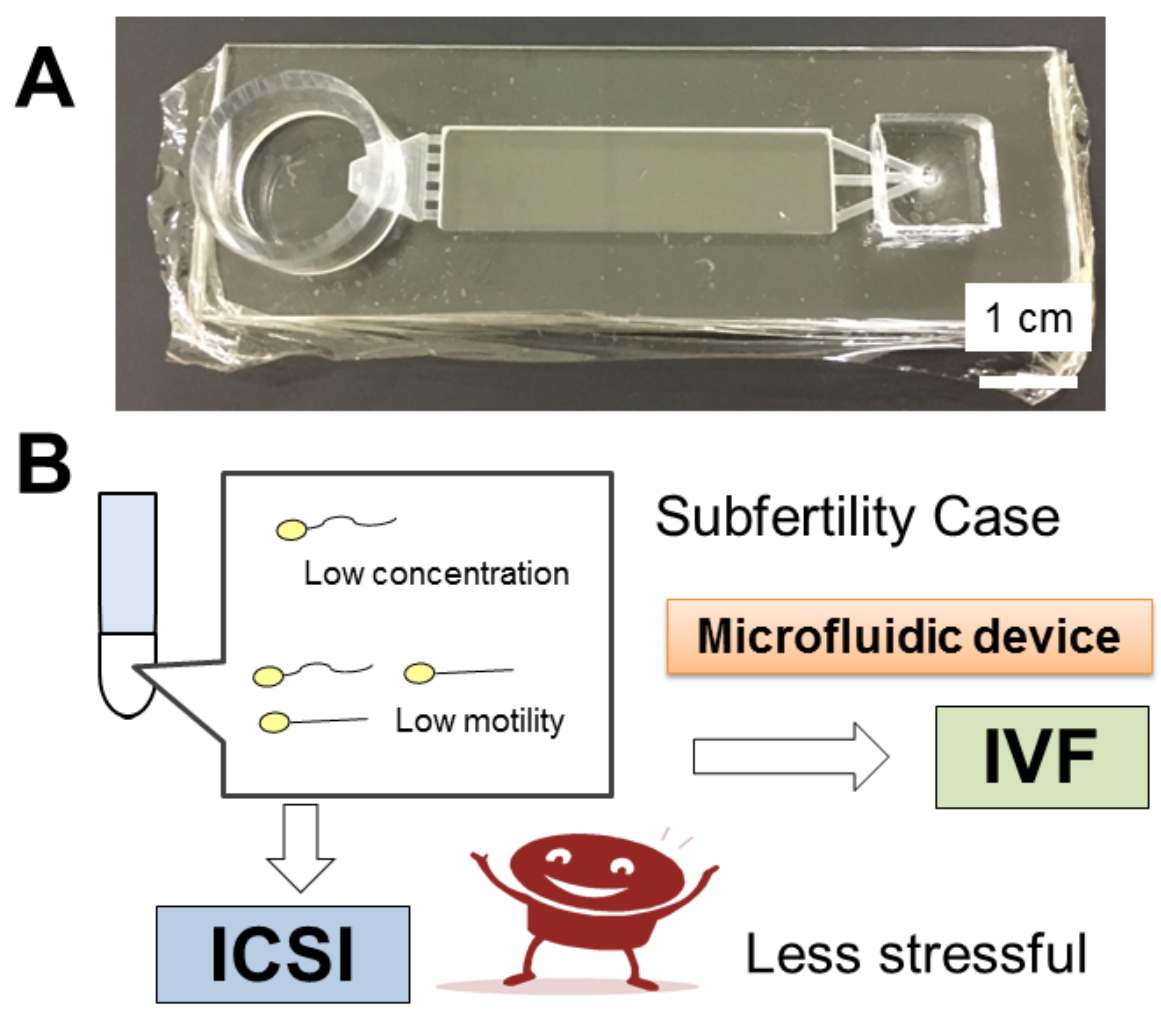

Figure 4. Microfluidic device for increasing sperm concentration in patients with oligospermia; (A) Image of the device; (B) Concept schematics of the benefits of this device for ART.

Plastic microfluidic devices have also been considered for increasing sperm concentration in cases of male infertility with oligospermia. Sperm collection for conventional ICSI methods is time consuming for samples with low sperm concentrations. Hence, if sperm counts can be increased using a microfluidic device, the duration and stress of ICSI will be reduced. To reduce ICSI treatment times for severe conditions, Matsuura etal. proposed a sperm 
collection method using a microfluidic chip device that can facilitate sperm observation without the use of centrifugation [64]. We compared the ICSI treatment times required for intracytoplasmic injection of porcine sperm using the microfluidic device method and the conventional microdroplet method before conducting clinical ART.
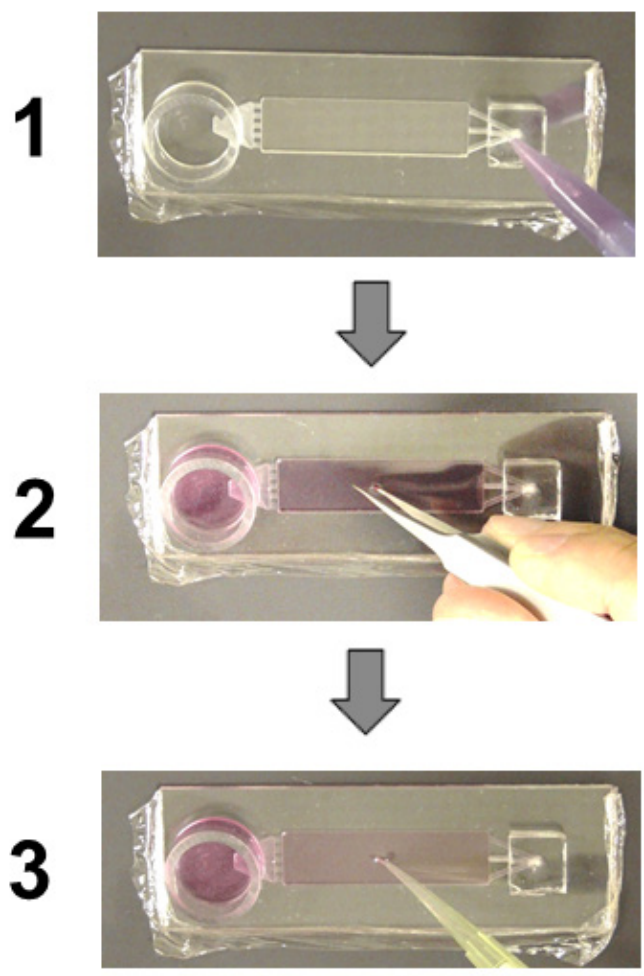

Figure 5. Protocol for increasing sperm concentration using the device; Medium containing the sperm sample was injected into the flow channel using a micropipette (1), the upper film in the centre chamber was pricked (2) and sperm were recovered using a micropipette (3).

The average ICSI treatment time using this microfluidic method with poor-quality semen was shorter than that using the conventional microdroplet method. These results suggest the clinical utility of this device for ICSI in human ART laboratories (Figure 4).

Previously, we developed a microfluidic device that increases sperm counts by mechanically drilling polymer plates and laminating the films. This device worked particularly well for semen samples with extremely low sperm counts (Figure 4A) [65] because sperm are concentrated in the centre chamber of the device within $30 \mathrm{~s}$ (Figure 5). Moreover, in comparison with conventional 
methods, no significant differences in treatment times were observed when semen was diluted to a sperm count of 100,000 cells $\mathrm{mL}^{-1}$. However, when the sperm count was adjusted to 10,000 cells $\mathrm{mL}^{-1}$, treatment times differed significantly between the two methods [64], suggesting that the microfluidic device could reduce the ICSI treatment time for semen samples with low sperm counts.

In further studies, changes in the height of the centre chamber and differences in shapes and diameters of microparticles (including human sperm) were investigated [65]. Specifically, we compared differences in concentrations using chip devices with heights of $1.0,1.4$ and $2.0 \mathrm{~mm}$ in the centre chamber. Diluted semen containing sperm with 10,000 cells $\mathrm{mL}^{-1}$ were most efficiently concentrated at a height of $1.4 \mathrm{~mm}$, whereas application of 100,000 cells $\mathrm{mL}^{-1}$ led to approximately 2 -fold increases in counts. Hence, this microfluidic device can increase sperm counts by approximately 10 -fold, allowing the option of IVF for patients who chose ICSI. These developments may improve fertility and sterility rates due to reduced stress levels of embryologists and reduced treatment costs. In future clinical studies, we will further validate and improve this technique.

\subsection{PAPER-BASED DEVICES FOR SEMEN DIAGNOSIS}

During the present decade, colorimetric and electrochemical paper-based POC devices have been proposed for many fields of diagnosis [66-68]. In particular, hydrophilic microfluidic channels were prepared using photolithography, wax printing, and screen printing, and the patterning technique may increase the potential applications of paper-based devices [66-68]. Andrological analyses of sperm motility and DNA integrity using paper-based devices have been suggested. Among these, Spermcheck is a currently marketed paper-based device for at-home fertility checking [69], and comprises a low-cost two-strip lateral flow immune-chromatographic diagnostic device that allows men to evaluate their sperm count privately [69]. Herr et al. demonstrated a direct relationship between sperm numbers and signal strengths in ELISA assays of SP-10 concentrations [70]. Based on these experimental results, the commercial immunodiagnostic device was successfully released using the ACRV1 protein analyte. In addition, blood-based diagnostics for male infertility are under evaluation based on ELISA [71-72]. However, the instability and expense of ELISA antibodies warrant further development of colorimetric metabolic assays of target cells as alternative diagnostic strategies.

To estimate sperm motility in semen in resource-limited settings, Matsuura et al. developed a 2-(4,5-dimethyl-2-thiazolyl)-3,5-diphenyl-2H-tetrazolium bromide (MTT) paper detection system that was based on energy metabolism [73-76]. Figure 6 shows the patterned paper device that was prepared by printing a filter paper using a wax printer and illustrates the mechanism of 
colour change. These authors compared sperm motility using CASA and calculated parameters of enzymatic reactivity in sperm. Sperm in semen moved on hydrophilic channels and did not penetrate the black wax. Subsequently, sperm evaluations were performed by applying semen onto the paper-based device treated with yellow MTT. Subsequently, catalytic sperm redox enzymes convert yellow MTT to the purple formazan metabolite [77-79]. Hence, the development of purple colour (8-bit grey scale converted) verifies enzyme activity and sperm motility. In this assay, small semen samples are placed on the paper test and the colour of semen spot on the paper changes after 30 min. Hence, the paper pattern can be recorded using a digital camera or a smartphone, allowing remote determinations of sperm counts and motility in semen.

To develop a model of non-motile sperm, we inactivated enzymes that are related to the motility of sperm samples by incubating human sperm at $50{ }^{\circ} \mathrm{C}$. Subsequently, we used the paper-based device to distinguish between sperm samples with greater than $50 \%$ motility and less than $10 \%$ motility by comparing average values from purple colour intensities. In further studies, sodium 2,3-bis(2-methoxy-4-nitro-5-sulfophenyl)-5-[(phenylamino)-carbonyl]-2H-tetrazolium inner salt (XTT) and sodium 5-(2,4-disulfophenyl)-2-(4-iodophenyl)-3-(4-nitrophenyl)-2Htetrazolium inner salt (WST-1) were applied to the paper-based device for sperm motility assays. However, MTT-treated patterned paper was the most suitable and cost-effective device [74]. We also found differing sensitivities of colorimetric motility parameters between porcine and human sperm [75], likely reflecting differences between molecular mechanisms of sperm motility. Further analyses of the molecular mechanisms of paper-based MTT assays are currently underway.

In a previous study, we compared colour intensities of MTT reduction signals in semen from healthy men and infertility patients [76]. After preservation at $37^{\circ} \mathrm{C}$, no significant differences in in motility parameters (MOTs) related with motlie sperm concentration were observed [76]. However, following storage at room temperature, MOTs of more than 100 million spermatozoa and more than 50 million motile spermatozoa were significantly increased. Similarly, Nosrati et al. reported a high correlation between MTT colour intensity changes on their paper-based device and motility of human semen in their clinical study [80]. Based on the results of these clinical studies, this assay can be used to determine numbers of motile sperm, and the results can be presented on a smartphone window in situ. Furthermore, this device can be used to predict possible risks of male infertility without directly observing sperm. These data warrant a multi-centre study of this paper-based device in routine use. 

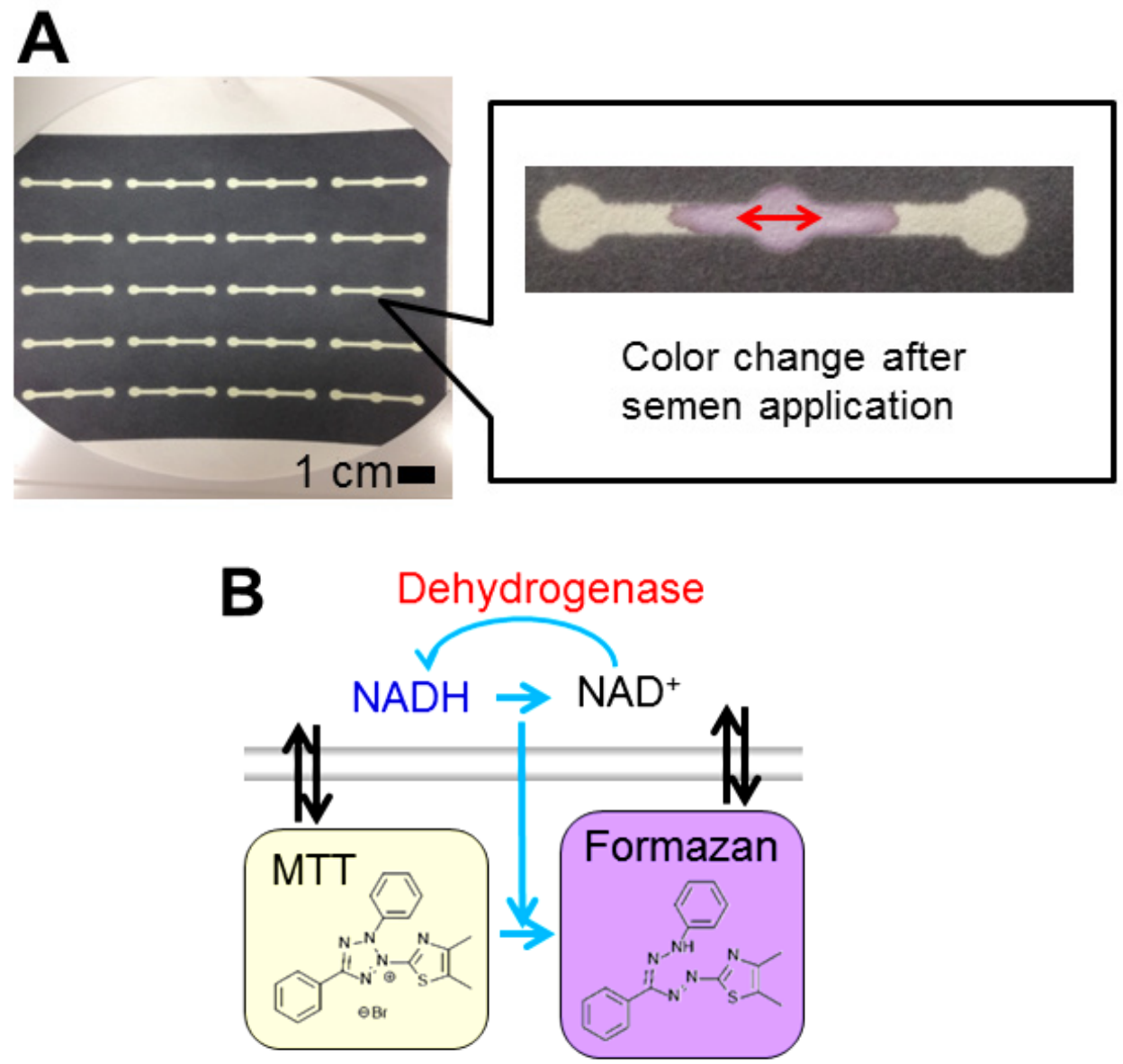

Figure 6. Paper-based device for sperm motility analysis using sperm enzyme reactions; (A) Paper-based device after MTT application; (B) Mechanism of purple colour change on this device.

In addition to sperm motility and morphology, DNA integrity is an important indicator of human fertility. Current representative DNA fragmentation analyses include Comet assays (single-cell gel electrophoresis), terminal deoxynucleotidyl transferase (TdT)-mediated dUDP nick-end labelling assays (TUNEL), Halo assays and sperm chromatin structure assays (SCSA) $[81,82]$. In Halo assays, halo signals are derived from the sperm head and are observed in sperm with little DNA fragmentation because dispersion of intact DNA is restricted in agarose gels. Conversely, when sperm DNA is fragmented, halo signals are not detected due to diffusion of fragmented DNA. SCSA uses acridine orange (AO) as a DNA intercalator and flow cytometry for high-throughput analysis. After intercalation into double-stranded DNA, AO emits 
green fluorescence, whereas single-strand DNA-AO conjugates (sometimes denatured DNA) have red fluorescence. Hence, comparisons of green-red fluorescence intensities indicate DNA fragmentation and are expressed using the DNA fragmentation index (DFI) [81,83]. Durations and reagents required for DNA integrity tests are summarized in Table 1 , and indicate that microfluidic techniques can reduce durations and reagent costs in assessments of sperm DNA integrity.

Table 1. Durations and costs of DNA integrity tests

\begin{tabular}{ccccc}
\hline & Duration & Reagents & $\begin{array}{c}\text { Instruments and } \\
\text { comments }\end{array}$ & Refs \\
\hline Comet & $\sim 120$ min & DNA staining dyes & $\begin{array}{c}\text { Electrophoresis device } \\
\text { and fluorescence } \\
\text { microscope }\end{array}$ & 87 \\
\hline TUNEL & $\sim 240$ min & $\begin{array}{c}\text { dUDP nick-end } \\
\text { labelling reagents }\end{array}$ & $\begin{array}{c}\text { Fluorescence } \\
\text { microscope or flow- } \\
\text { cytometer }\end{array}$ & 88 \\
\hline $\begin{array}{c}\text { Halo (DNA } \\
\text { dispersion } \\
\text { test) }\end{array}$ & $120 \mathrm{~min}$ & $\begin{array}{c}\text { DNA staining dyes } \\
\text { and agarose }\end{array}$ & $\begin{array}{c}\text { Fluorescence } \\
\text { microscope }\end{array}$ & 89 \\
\hline SCSA & $20 \mathrm{~min}$ & Acridine orange & Flow cytometer & 82 \\
\hline $\begin{array}{c}\text { Silicone-based } \\
\text { microfluidics }\end{array}$ & $20 \mathrm{~min}$ & LIVE/DEAD sperm \\
viability kit & $\begin{array}{c}\text { Fluorescence } \\
\text { microscope and silicone } \\
\text { or plastic microfluidic } \\
\text { channel }\end{array}$ & 84 \\
\hline $\begin{array}{c}\text { Paper-based } \\
\text { test }\end{array}$ & 15 min & Acridine orange & $\begin{array}{c}\text { Fluorescence } \\
\text { microscope }\end{array}$ & 85 \\
\hline
\end{tabular}

SCSA and all other methods require high-cost flow cytometry and fluorescence microscopy, respectively, whereas fluorescence microscopy-based DNA fragmentation assays using a smartphone could be applied in the future. Relationships between DNA fragmentation and fertility have been discussed previously [82,84], and in a previous meta-analysis by Osman et al., significant increases in live birth rates were observed after fertilization with sperm that had lower DNA fragmentation rates [82]. Moreover, using silicone microfluidic channels, motile sperm with high DNA integrity were selected toward a central outlet based on progressive motility in 500 parallel microchannels with cross-section dimensions of $100 \times 75 \mu \mathrm{m}$ [85]. In 2016, Nosrati et al. reported a novel analytical system for assessing DNA integrity using ion concentration polarization on paper and applying voltage $(150 \mathrm{~V})$ to the sample channel on the paper [86]. These investigators suggested that SCSA offers high through put, but high cost assessments of DNA fragmentation. Accordingly, paper- 
-based sperm DNA integrity tests can reduce durations and costs, and are more suitable for use in resource-limited or remote settings.

\subsection{DISCUSSION AND SATISFACTION OF CRITERIA FOR SEMEN CHARACTERISTICS}

Herein, we introduced smartphone-based cell detection devices, plastic-based microfluidic devices for infertility treatments and plastic- or paper-based microfluidic devices for semen diagnoses. In sections 4.4. and 4.5., we summarized evidence that sorted motile sperm possess higher DNA integrity, as indicated by studies of microfluidic devices without centrifugation $[57,58,62,63,85]$. Developments and applications of microfluidics have contributed markedly to improvements in ART over the past 20 years. However, LCIVF treatment protocols for male infertility have not been standardized in developing countries. Obstacles for the uptake of low-cost diagnostic and treatment devices in developing countries include usability and non-actualized costs. Although penetration of smartphone devices has been rapid, marketing of materials and devices has been slow. However, these novel devices should be recognized by users after reliable clinical studies.

\subsection{CONCLUSION}

The present plastic- and paper-based microfluidic devices have potential uses in IUI and IVF. In particular, sperm analyses that can be performed at home will reduce the cost of treatments. Hence, simple and efficient microfluidic devices and smartphone-based optical analysis devices will likely be adopted for routine clinical use after evaluation in ART laboratories. Clinical studies of these devices are urgently needed to accommodate increases in sperm sample numbers in the coming decade. Moreover, some novel microfluidic devices may be applied for routine clinical use, including in the LCIVF project. Finally, these low-cost sperm analysis devices can be used to determine sperm concentrations, motility and DNA integrity in men who do not have enough time to have their sperm characteristics examined at the clinic.

\section{ACKNOWLEDGEMENTS}

This study was supported by a grant-in-aid for Scientific Research for Fundamental Sciences (Nos. 26350106 and 26220203 to K.M. as principal and supporting investigator, respectively) from The Ministry of Education, Culture, Sports, Science and Technology (MEXT) of Japan. The authors would like to thank Enago for the English language review. 


\section{REFERENCES}

1. A. Agarwal, A. Mulgund, A. Hamada, M.R. Chyatte. Reprod. Biol. Endocrinol. 13 37 (2015) 9 pages.

2. $\quad$ N. Kumar, A.K. Singh. J. Hum. Reprod. Sci. 8 (2015) 191-196.

3. L. Stuppia, M. Franzago, P. Ballerini, V. Gatta, I. Antonucci. Clin. Epigenetics 120 (2015) 15 pages.

4. $\quad$ M.C. Inhorn, P. Patrizio. Hum. Reprod. Update 21 (2015) 411-426.

5. W. Ombelet. FVV Obgyn (2012) Monograph: 7-16.

6. T.K. Aleyamma, M.S. Kamath, K. Muthukumar, A.M. Mangalaraj, K. George. Hum. Reprod. 26 (2011) 3312-3318.

7. S.C. Esteves, A. Zini, N. Aziz, J.G. Alvarez, E.S. Sabanegh, A. Agarwal. Urology 79 (2012) 16-22.

8. W. Ombelet, N. Dhont, A.Thijssen, E. Bosmans, T. Kruger. Reprod. BioMed. Online 28 (2014) 300-309.

9. $\quad$ B.D. Anawalt. J. Clin. Endocrinol. Metab. 98 (2013) 3532-3542.

10. M. van Wely, N. Barbey, A. Meissner, S. Repping, S.J. Silber. Hum. Reprod. 30 (2015) 1-6.

11. H.W. Jones, I. Cooke, R. Kempers, P. Brinsden, Saunders D. International Federation of Fertility Societies: Surveillance 2010, http://iffsreproduction.org/documents/IFFS_Surveillance_2010.pdf $(1 / 10 / 2016)$

12. W. Ombelet. Reprod. Biomed. Online 28 (2014) 267-272.

13. G. Pennings, G. de Wert, F. Shenfield, J. Cohen, B. Tarlatzis, P. Devroey. Hum. Reprod. 24 (2009) 1008- 1011.

14. P. Devroey, B.C.J.M. Fauser, K. Diedrich, on behalf of the Evian Annual Reproduction (EVAR) Workshop Group (2008). Hum. Reprod. Update 15 (2009) 391-408.

15. M. Kakihara, Grasping a Global View of Smartphone Diffusion: An Analysis from a Global Smartphone Study, International Conference on Mobile Business. Paper 11 (2014).

16. M.R. Islam, M.R. Islam, T.A. Mazumder. Int. J. Eng. Tech. 10 (2010) No 06.

17. J. Hu, X. Cui, Y. Gong, X. Xu, B. Gao, T. Wen, T.J. Lu, F. Xu. Biotech. Adv. 34 (2016) 305-320.

18. C.J. Tuijn, B.J. Hoefman, H. van Beijma, L. Oskam, N. Chevrollier. PLoS One 6 (2011) e28348.

19. Q. Wei, H. Qi, W. Luo, D. Tseng, S.J. Ki, Z. Wan, Z. Gorocs, L.A. Bentolila, T. Wu, R. Sun, A. Ozcan. ACS Nano 7 (2013) 9147-9155.

20. M. Fraczek, E. Wiland, M. Piasecka, M. Boksa, D. Gaczarzewicz, A. SzumalaKakol, T. Kolanowski, L. Beutin, M. Kurpisz. Fertil. Steril. 102 (2014) 711-719.

21. M. Punab, K. Lõivukene, K. Kermes, R. Mändar. Andrologia 35 (2003) 271-278.

22. E. Moretti, S. Capitani, N. Figura, A. Pammolli, M.G. Federico, V. Giannerini, G. Collodel. J. Assist. Reprod. Genet. 26 (2009) 47-56.

23. S. La Vignera, R.A. Condorelli, E. Vicari, M. Salmeri, G. Morgia, V. Favilla, S. Cimino, A.E. Calogero. J. Med. Microbiol. 63 (2014) 1-14.

24. J.R. Hutchison, R.L. Erikson, A.M. Sheen, R.M. Ozanich, R.T. Kelly. Analyst 140 (2015) 6269-6276.

25. T.S. Park, W. Li, K.E. McCracken, J.Y. Yoon. Lab Chip 13 (2013) 4832-4840.

26. C. Garrett, D.Y. Liu, G.N. Clarke, D.D. Rushford, H.W. G. Baker. Hum. Reprod. 18 (2003) 1643-1649. 
27. S.T. Mortimer, G. van der Horst, D. Mortimer. Asian J. Androl. 17 (2015) 545-553.

28. D. Breslauer, R. Maamari, N. Switz, W. Lam, D.A. Fletcher. PLoS One 4 (2009) e6320.

29. T.-W. Su, A. Erlinger, D. Tseng, A. Ozcan. Anal. Chem. 82 (2010) 8307-8312.

30. T.-W. Su, L. Xue, A. Ozcan. Proc. Natl. Acad. Sci. 109 (2012) 16018-16022.

31. X. Zhang, I. Khimji, U.A. Gurkan, H. Safaee, P.N. Catalano, H.O. Keles, E. Kayaalp, U. Demirci. Lab Chip 11 (2011) 2535-2540.

32. H. Im, C.M. Castro, H. Shao, M. Liong, J. Song, D. Pathania, L. Fexon, C. Min, M. Avila-Wallace, O. Zurkiya, J. Rho, B. Magaoay, R.H. Tambouret, M. Pivovarov, R. Weissleder, H. Lee. Proc. Natl. Acad. Sci. 112 (2015) 5613-5618.

33. Y. Kobori, P. Pfanner, G.S. Prins, C. Niederberger. Fertil. Steril. 106 (2016) 574-578.

34. 'iSperm' http://isperm.aidmics.com/ (1/10/2016).

35. C.D. Chin, V. Linder, S.K. Sia. Lab Chip 12 (2012) 2118-2134.

36. V. Gubala, L.F. Harris, A.J. Ricco, M.X. Tan, D.E. Williams. Anal. Chem. 84 (2012) 487-515.

37. P.B. Luppa, A. Bietenbeck, C. Beaudoin, A. Giannetti. Biotech. Adv. (2016) web published.

38. S. Patel, R. Nanda, S. Sahoo, E. Mohapatra. Biochem. Res. Int. (2016) 3130469.

39. L.J. Kricka, I. Faro, S. Heyner, W.T. Garside, G. Fitzpatrick, G. McKinnon, J. Ho, P. Wilding. J. Pharm. Biomed. Anal. 15 (1997) 1443-1447.

40. K. Matsuura, K. Naruse, Advanced elastomers: technology, properties and applications, InTech, Rijeka, 2012, p. 243-262.

41. S.M. Knowlton, M. Sadasivam, S. Tasoglu. Trends Biotech. 33 (2015) 221-229.

42. S.G. Clark, K. Haubert, D.J. Beebe, C.E. Ferguson, M.B. Wheeler. Lab Chip 5 (2005) 1229-1232.

43. R.S. Suh, X. Zhu, N. Phadke, D.A. Ohl, S. Takayama, G.D. Smith. Hum. Reprod. 21 (2006) 477-483.

44. C. Han, Q. Zhang, R. Ma, L. Xie, T. Qiu, L. Wang, K. Mitchelson, J. Wang, G. Huang, J. Qiao, J. Cheng. Lab Chip 10 (2010) 2848-2854.

45. K. Matsuura, Y. Asano, K. Naruse, Recent Advances in Mechanobiology, Shanghai scientific and technological literature publishing house, Shanghai, China, 2012, p. 161-166.

46. R. Ma, L. Xie, C. Han, K. Su, T. Qiu, L. Wang, G. Huang, W. Xing, J. Qiao, J. Wang, J. Cheng. Anal. Chem. 83 (2011) 2964-2970.

47. M.C. McCormack, S. McCallum, B. Behr. J. Urol. 175 (2006) 2223-2227.

48. L.I. Segerink, A.J. Sprenkels, P.M. ter Braak, I. Vermes, A. van den Berg. Lab Chip 10 (2010) 1018-1024.

49. S. Tasoglu, H. Safaee, X. Zhang, J.L. Kingsley, P.N. Catalano, U.A. Gurkan, A. Nureddin, E. Kayaalp, R.M. Anchan, R.L. Maas, E. Tüzel, U. Demirci. Small 9 (2013) 3374-3384.

50. Y.A. Chen, Z.W. Huang, F.S. Tsai, C.Y. Chen, C.M. Lin, A.M. Wo. Microfluid. Nanofluid. 10 (2011) 59-67.

51. C.-Y. Chen, T.-C. Chiang, C.-M. Lin, S.-S. Lin, D.-S. Jong, V.F.-S. Tsai, J.-T. Hsieh, A. M. Wo. Analyst 138 (2013) 4967-4974.

52. 'Protocol of Miclofluidic Sperm Sorter in Japanese' http://meniconlifescience.com/pdf/ssq-guide.pdf (1/10/2016).

53. J.E. Swain, D. Lai, S. Takayama, G.D. Smith. Lab Chip 13 (2013) 1213-1224. 
54. B.S. Cho, T.G. Schuster, X. Zhu, D. Chang, G.D. Smith, S. Takayama. Anal. Chem. 75 (2003) 1671-1675.

55. T.G. Schuster, B.S. Cho, L. Keller, S. Takayama, G.D. Smith. Reprod. BioMed. Online 7 (2003) 75-81.

56. J.M. Wu, Y. Chung, K.J. Belford, G.D. Smith, S. Takayama, J. Lahann. Biomed. Microdevices 8 (2006) 99-107.

57. R.T. Schulte, Y.K. Chung, D.A. Ohl, S. Takayama, G.D. Smith. Fertil. Steril. 88 (2007) S76.

58. D. Shibata, H. Ando, A. Iwase, T. Harata, F. Kikkawa, K. Naruse. Fertil. Steril. 88 (2007) S110.

59. H. Sano, K. Matsuura, K. Naruse, H. Funahashi. Thriogenelogy 74 (2010) 863-870.

60. K. Matsuura, M. Takenami, Y. Kuroda, T. Hyakutake, S. Yanase, K. Naruse. Reprod. Biomed. Online 24 (2012) 109-115.

61. K. Shirota, F. Yotsumoto, H. Itoh, H. Obama, N. Hidaka, K. Nakajima, S. Miyamoto. Fertil. Steril. 105 (2016) 315-321.

62. W. Asghar, V. Velasco, J.L. Kingsley, M.S. Shoukat, H. Shafiee, R.M. Anchan, G.L. Mutter, E. Tüzel, U. Demirci. Adv. Healthc. Mater. 3 (2014) 1671-1679.

63. T. Chinnasamy, B. Behr, U. Demirci. Fertil. Steril. 105 (2016) Supplement, e17-e18.

64. K. Matsuura, T. Uozumi, T. Furuichi, I. Sugimoto, M. Kodama, H. Funahashi. Fertil. Steril. 99 (2013) 400-407.

65. K. Matsuura, K. Nishida, I. Sugimoto, S. Yanase. Androl. Gynecol.: Curr. Res 1 (2013) 1-3.

66. E.W. Nery, L.T. Kubota. Anal. Bioanal. Chem. 405 (2013) 7573-7595.

67. J. Hu, S.-Q. Wang, L. Wang, F. Li, B. Pingguan-Murphy, T.J. Lu, F. Xu. Biosens. Bioelectron. 54 (2014) 585-597.

68. D.M. Cate, J.A. Adkins, J. Mettakoonpitak, C.S. Henry. Anal. Chem. 87 (2015) 19-41.

69. M.A. Coppola, K.L. Klotz, K. Kim, H.Y. Cho, J. Kang, J. Shetty, S.S. Howards, C.J. Flickinger, J.C. Herr. Hum. Reprod. 25 (2010) 853-861.

70. J.C. Herr, K.L. Klotz, P. Anderson, E. Adams, N. Moore, S. Howards. Clin. Immunol. Newsl. 19 (1999) 52-58.

71. J.M. Bieniek, A.P. Drabovich, K.C. Lo. Asian J. Androl. 18 (2016) 426-433.

72. K.L. Klotz, M.A. Coppola, M. Labrecque, V.M. Brugh III, K. Ramsey, K. Kimg, M.R. Conawayb, S.S. Howardsc, C.J. Flickingera, J.C. Herr. J. Urol. 180 (2008) 2569-2576.

73. K. Matsuura, K.-H. Chen, C.-H. Tsai, W.-Q. Li, Y. Asano, K. Naruse, C.-M. Cheng. Microfluid. Nanofluid. 14 (2014) 1378-1388.

74. K. Matsuura, K.-H. Chen, C.-H. Tsai, W.-Q. Li, Y. Asano, K. Naruse, C.-M. Cheng. Japanese Soc. Med. Biol. Eng. 52 (2014) 0-551-552.

75. W.-Q. Li, K. Matsuura, K.-H. Chen, Y. Asano, K. Naruse, C.-M. Cheng, IEEE NANOMED, Porcine and Human Sperm Motility Analyses Using Paper-based Diagnostic Devices, Kaohsiung, Taiwan, 2014.

76. K. Matsuura, J. Komiyama, O. Okitsu. Fertil. Steril. 104 (2015) e239.

77. M.V. Berridge, P.M. Herst, A.S. Tan. Biotech. Annual Rev. 11 (2005)127-152.

78. B.M. Berg. Open Veterinary J. 5 (2015) 58-63.

79. M.H. Nasr-Esfahani, R. Aboutorabi, E. Esfandiari, M. Mardani. J. Assist. Reprod. Genet. 19 (2002) 477-482. 
80. R. Nosrati, M.M. Gong, M.C. San Gabriel, C.E. Pedraza, A. Zini, D. Sinton. Clinic. Chem. 62 (2016) 458-465.

81. S.E.M. Lewis, R.J. Aitken, S.J. Conner, G. De Iuliis, D.P. Evenson, R. Henkel, A. Giwercman, P. Gharagozloo. Reprod. BioMed. Online 27 (2013) 325-337.

82. A. Osman, H. Alsomait, S. Seshadri, T. El-Toukhy, Y. Khalaf. Reprod. BioMed. Online (2015) 30 120-127.

83. D.P.Evenson, L.K. Jost, D. Marshall, M.J. Zinaman, E. Clegg, K. Purvis, P. de Angelis, O.P. Claussen. Hum. Reprod. 14 (1999) 1039-1049.

84. M. Muratori, S. Marchiani, L. Tamburrino, M. Cambi, F. Lotti, I. Natali, E. Filimberti, I. Noci, G. Forti, M. Maggi, E. Baldi. Fertil. Steril. 104 (2015) 582-590.

85. R. Nosrati, M. Vollmer, L. Eamer, M.C. San Gabriel, K. Zeidan, A. Zini, D. Sinton. Lab Chip 14 (2014) 1142-1150.

86. R. Nosrati, M.M. Gong, M.C.S. Gabriel, A. Zini, D. Sinton. Anal. Methods 8 (2016) 6260-6264.

87. G.A. Haines, J.H. Hendry, C.P. Daniel, I.D. Morris. Biol. Reprod. 67 (2002) 854-861.

88. M. Sergerie, G. Laforest, K. Boulanger, F. Bissonnette, G. Bleau. Hum. Reprod. 20 (2005) 1921-1927.

89. L. Muriel, M. Meseguer, J.L. Fernández, J. Alvarez, J. Remohí, A. Pellicer, N. Garrido. Hum. Reprod. 21 (2006) 738-744. 